\title{
RF Calibration of On-Chip DfT Chain by DC Stimuli and Statistical Multivariate Regression Technique
}

\author{
Rashad Ramzan and Jerzy Dabrowski
}

\section{Linköping University Post Print}

\section{Tweet}

N.B.: When citing this work, cite the original article.

Original Publication:

Rashad Ramzan and Jerzy Dabrowski, RF Calibration of On-Chip DfT Chain by DC Stimuli and Statistical Multivariate Regression Technique, 2015, Integration, (49), 14-21.

http://dx.doi.org/10.1016/j.vlsi.2014.11.006

Copyright: Elsevier

http://www.elsevier.com/

Postprint available at: Linköping University Electronic Press

http://urn.kb.se/resolve?urn=urn:nbn:se:liu:diva-105514 


\section{Comparisons of automated blood pressures in a primary health care setting with self-measurements at the office and at home using the Omron i-C10 device}

Isam Al-Karkhi", Raad Al-Rubaiy*, Ulf Rosenqvist, Magnus Falk, Fredrik H Nystrom

Department of Medical and Health Sciences, Faculty of Health Sciences, Linköping University, Linköping, Sweden. * These authors contributed equally to the study.

Corresponding author:

Fredrik H. Nystrom M.D., Ph.D., professor

Department of Medical and Health Sciences

Linköping University

SE 58185 Linköping, Sweden

Telephone; +46101037749

Fax; +4613 145949

E-mail; fredrik.nystrom@lio.se 


\begin{abstract}
Objective We aimed to compare blood pressure (BP) levels recorded using the semiautomatic oscillometric Omron i-C10 blood pressure device in patients with or without hypertension in three different settings: 1 , when used by a doctor or a nurse at the office (OBP); 2, when used for self-measurement by the patient at the office (SMOBP); 3, when used for 7 consecutive days at home (HBP).

Methods A total of 247 subjects were invited to participate but 78 of these declined and a further 7 were excluded, leaving a final cohort of 162 participants.

Results Mean OBP was higher than HBP (difference 8.1 $\pm 14 / 3.1 \pm 8.8 \mathrm{mmHg}$, $\mathrm{p}<0.0001$ ) and so was SMOBP compared with HBP (difference 7.0 $\pm 13 / 4.2 \pm 7.3$ $\mathrm{mmHg}, \mathrm{p}<0.0001)$. Sixteen participants $(9.9 \%)$ had $\geq 10 \mathrm{mmHg}$ higher systolic SMOBP than OBP and $28(17 \%)$ participants had $\geq 10 \mathrm{mmHg}$ lower systolic SMOBP than OBP. Subjects that were current smokers had a larger mean difference between systolic OBP and SMOBP than non-smokers (OBP - SMOBP in smokers: $6.6 \pm 9.4$ mmHg, OBP - SMOBP in non-smokers: $0.5 \pm 9.2 \mathrm{mmHg}, \mathrm{p}=0.011$ between groups) Conclusion Self-measurement of BP in the office does not preclude an increase in BP when levels in the individual patients are compared with HBP using the same equipment. Thus SMOBP with a semi-automatic device does not achieve the reduction of the white-coat effect in the same manner as fully automatic devices.
\end{abstract}

Key words Home blood pressure, hypertension, office blood pressure, smoking, oscillometric.

\title{
Introduction
}

Many patients temporarily increase blood pressure (BP) levels at the office when this 
is recorded by a doctor or a nurse [1,2]. Accordingly ambulatory blood pressure recordings or self-measurement of blood pressure at home (HBP) is often recommended for a final diagnosis of hypertension when there is suspicion of this so called "white-coat effect" of the office blood pressure recording (OBP) [3, 4]. It is not entirely settled whether the increase in office blood pressure is induced by presence of the provider of care, the nurse or doctor, or whether it is the setting that is most important. However, studies using the BpTRU monitor have shown that selfmeasurement of BP at the office by the patient gives mean BP that are similar to those measured as awake ambulatory BP suggestive of that the white-coat effect is eliminated $[5,6]$. In Canada it is quite prevalent to use the fully automatic BpTRU equipment for BP recordings at the office in routine care [6]. In Sweden, on the other hand, many primary health care centers are using traditional semi-automatic home BP monitors that are considerably cheaper than the BpTRU monitor for the same purpose. These measurements are usually undertaken in dedicated "self-health care rooms" in which the patients measure the BP without supervision, but instructed to rest alone for 5 minutes ahead of BP recordings, and then to leave a note with the actual BP measured to the provider of care, for a diagnosis or for the follow-up of hypertension. This supposedly saves time for the patient and also costs for the primary health care center since no personnel are needed for the BP measurement. Furthermore, the patients does not need to book any particular appointment for the blood pressure measurement. We know of only one dedicated study that has compared self-measurement of blood pressure at the office (SMOBP) with blood pressure recordings by the provider of care using the same equipment when this is done with a semi-automatic oscillometric automated blood pressure device, that has been validated and found to provide blood pressure recordings with high quality [7]. 
However since this earlier study was of a limited size, $n=30$, detailed analyses of potential confounders of differences between OBP and SMOBP were challenging [7].

We aimed to investigate and compare BP levels recorded with the same oscillometric BP recorder, the Omron i-C10 blood pressure device (derivative of Omron M-7), in patients with or without known hypertension, in three different settings: 1 , when used by a doctor or a nurse at the office; 2 , when used for self-measurement by the patient

at the health care center; 3 , when used for 7 consecutive days for self-measurement of $\mathrm{BP}$ at home. We wanted not only to compare the mean values at the different settings and correlations between recordings but also to study if there were any more systematic interactions between differences in BP levels measured in the three settings and anthropometrics or risk factors for cardiovascular disease. In contrast to most earlier studies [7,8], our cohort included patients with and without established hypertension. The study aimed to investigate whether conventional manual BP can be replaced with use of a semi-automatic home BP recorder in order to obtain readings which are similar to the HBP and less affected by the white-coat effect associated with conventional manual BP.

\section{Materials and methods}

The study was conducted in two primary health care centers in the city of Motala which is located in southeastern Sweden. The subjects were invited to participate in the blood pressure study between June and October of 2013. The invitation was provided either during a regular visit to the primary health care center or by a letter to patients with an established diagnosis of hypertension. Exclusion criteria were atrial fibrillation and recent start ( $<3$ months) of treatment with antihypertensive drugs in 
order to reduce BP in hypertension. A questionnaire was filled out by each participant including information on smoking, concomitant diseases and medications.

Participants were randomly assigned to one out of two groups, one in which the subjects began with blood pressures measurement at the health care center by a doctor or a nurse, and one in which the participants began with SMOBP in the designated room at the health care center. A semi-automatic Omron i-C10 Blood Pressure Monitor was used for all blood pressure measurements at the offices and at home.

All blood pressure measurements in the office and at home were done after 5 minutes of rest. The first measurements, were performed three times in each arm and the arm with the highest blood pressure (any difference) was then used for subsequent recordings at office and home blood pressure measurements. There was a one minute interval between the three blood pressure recordings performed. For the selfmeasurements the patients were alone without any health professional in the room and after 5 minutes of rest, seated with bilateral armrests, and the upper arm in which blood pressure was measured to be positioned at heart level, they started the semiautomatic device for the three recordings. At home the blood pressure measurements were performed by the patients in the same manner and arm as at the office, in the morning and in the evening, for seven consecutive days. The results of the recordings were noted on a dedicated sheet of paper by the patients. The mean value of the last two measurements on each blood pressure recording (at the office and at home in both the morning and evening) were calculated and used in the results presented herein. Thus HBP consisted of $2+2$ recordings on each day (morning and evening) for 7 days, i.e. a total of $28 \mathrm{BP}$ measurements. 


\section{Ethics}

All participants gave written informed consent prior to participating in the study. The study, which complied with the declaration of Helsinki, was approved by the Regional Ethical Review Board in Linköping, Sweden.

\section{Statistics}

IBM SPSS statistics 21 and 22 (IBM corporation, Somers, NY, USA) were used for statistics. Linear correlations were calculated, comparisons within and between groups were done with the Student paired and unpaired two-tailed t test. Mean (SD) is given unless otherwise stated. Statistical significance was considered at the 5\% level $(\mathrm{p} \leq 0.05)$

\section{Results}

A total of 247 subjects were invited to participate but 78 of these declined and a further 7 were excluded based on study criteria, leaving a final cohort of 162 participants that successfully underwent and performed blood pressure measurements in the office, in the self-measurement room at the health care center and who also performed blood pressure recordings at home for seven days. No participants were lost to follow-up.

All participants had arm circumferences that allowed use of the standard blood pressure "one-size $22-42 \mathrm{~cm}$ " cuff, that has been validated for use also on large arms with circumferences from 32 to $42 \mathrm{~cm}[9,10]$. 
The first measured systolic BP was on average 1-2 mmHg higher than the following two BP measurements (comparison of all measurements, $\mathrm{p}<0.0001$ ) when the three $\mathrm{BP}$ were recorded by either OBP, SMOBP or HBP. The corresponding diastolic BP differences were numerically smaller, varying from $0.4-0.6 \mathrm{mmHg}(\mathrm{p}<0.0001)$. All data here are presented after disregarding this first recorded BP on each occasion, i.e. as mean values of the remaining two BP recordings.

Table 1 shows characteristics of the participants including mean BP levels at the three settings. Mean HBP levels were lower than both SMOBP and OBP, while mean OBP and SMOBP did not differ significantly (mean systolic difference $1.1 \pm 9.4 \mathrm{mmHg}$, $\mathrm{p}=0.14$, mean diastolic difference $-1.1 \pm 7.9 \mathrm{mmHg}, \mathrm{p}=0.085$, see Table 1$)$. There were strong correlations between SMOBP and OBP (systolic BP: $r=0.87, \mathrm{p}<0.0001$, Diastolic BP: $r=0.73, \mathrm{p}<0.0001$ ). OBP correlated with HBP (systolic BP: $r=0.69, \mathrm{p}<$ 0.0001, Diastolic BP: $\mathrm{r}=0.63, \mathrm{p}<0.0001)$ and correspondingly SMOBP correlated with HBP (systolic BP: $r=0.72, p<0.0001$, diastolic BP: $r=0.72, p<0.0001$ ).

Mean difference between OBP and HBP was $8.1 \pm 14 / 3.1 \pm 8.8 \mathrm{mmHg}(\mathrm{p}<0.0001$ for both systolic and diastolic levels) and corresponding levels of difference between SMOBP and HBP was $7.0 \pm 13 / 4.2 \pm 7.3 \mathrm{mmHg}(\mathrm{p}<0.0001$ for both systolic and diastolic levels). On an individual levels some patients showed large differences between BP measurements at the different settings, as shown by histograms for systolic OBP - SMOBP, OBP - HBP, and SMOBP - HBP (Figures 1 a-c). There were 16 participants $(9.9 \%)$ that had at least $10 \mathrm{mmHg}$ higher systolic SMOBP than OBP and conversely $28(17 \%)$ participants who had at least $10 \mathrm{mmHg}$ lower systolic SMOBP than OBP. 
There were no significant correlations between the differences of systolic BP measured at the three settings with BMI, gender, diagnosis of hypertension or age. However, the magnitude of the OBP - SMOBP difference correlated positively with OBP levels (systolic: $\mathrm{r}=0.18, \mathrm{p}=0.019$, diastolic $\mathrm{r}=0.45, \mathrm{p}<0.0001$ ) i.e. at higher OBP levels there was also a larger difference compared with SMOBP levels. Correspondingly there was a negative correlation between the OBP - SMOBP difference with SMOBP (systolic values $r=-0.33, p<0.0001$, diastolic values $r=-$ $0.28, \mathrm{p}<0.0001)$. Figure 2 shows the relationships between HBP and the SMOBP minus HBP difference and also that of HBP in relation to the OBP minus HBP difference, i.e. so called Bland-Altman plots.

Mean blood pressures at home or at the office did not differ when levels in current smokers $(n=17)$ were compared with non-smokers, but there was a non-significant trend for a higher mean systolic SMOBP in non-smokers than in smokers (smokers: $132.3 \pm 16 \mathrm{mmHg}$, non-smokers: $138.6 \pm 19 \mathrm{mmHg}, \mathrm{p}=0.19)$. Subjects that were current smokers had a larger mean difference between systolic OBP and SMOBP than non-smokers (OBP - SMOBP in smokers: $6.6 \pm 9.4 \mathrm{mmHg}$, OBP - SMOBP in nonsmokers: $0.5 \pm 9.2 \mathrm{mmHg}, \mathrm{p}=0.011$ between groups) while there was no such difference between OBP and HBP ( $\mathrm{p}=0.78$ between groups). Smokers had similar systolic self-measured BP at the office and at home while non-smokers had comparatively lower systolic HBP (SMOBP - HBP in smokers: $0.64 \pm 14 \mathrm{mmHg}$, SMOBP - HBP in non-smokers: $7.7 \pm 13 \mathrm{mmHg}, \mathrm{p}=0.034$ between groups). 
SMOBP in smokers (diastolic OBP - SMOBP in smokers: $3.8 \pm 4.6 \mathrm{mmHg}$, diastolic OBP - SMOBP in non-smokers $-1.7 \pm 8.0 \mathrm{mmHg}, \mathrm{p}=0.007$ between groups).

\section{Discussion}

By using the semi-automatic Omron i-C10 device we did not attain similar results of studies of SMOBP using the fully automatic BpTRU device, that the white-coat effect of being at the office is generally absent $[6,8]$. Rather our findings are similar to the first studies of SMOBP that found the setting to be of greater importance than whether the measurement is performed by the patient or by others $[7,11]$. Our findings are thus in line with the first specific investigations of the importance of the setting for BP levels when an Omron Hem 705CP was used [7], an apparatus very similar to the one used in our study. Stergiou et al. investigated the importance of the setting for the BP recordings and based on their findings, similar to ours, suggested that "home BP" is a more correct term than "self-measured BP" [7]. Stergiou et al. also demonstrated this by showing that self-measured BP in the home did not markedly differ from home BP recorded by a relative [7]. A finding that also suggests the importance of the setting as compared with the particular subject who records the BP.

In Canada the BpTRU device is commonly used for SMOBP and it has been showed to reduce the white-coat effect in the office when this has been compared with manual BP recordings [8]. This device is considerably more expensive than the Omron i-C10 we have used in our study, and we know of no study in which the BpTRU has been compared in different settings in a manner similar to ours. Although it is convenient in many ways to allow patients to visit the office and record BP using semi-automatic 
BP measuring equipment in a flexible manner without having to book an appointment, our findings suggest that this gives BP values that often differ considerably from those measured by a doctor or a nurse using the same technique for the actual BP recording. Thus our data suggest that manual OBP should preferably be replaced with fully automatic BP recordings and not semi-automatic SMOBP to achieve similar mean values as HBP.

Previous studies on the importance of the setting for self-measurement of BP has mainly focused on correlations and on mean values of BP levels in the different settings [8]. Although mean values of SMOBP and OBP did not differ in our study we found $26.9 \%$ of recorded systolic $\mathrm{BP}$ to differ $10 \mathrm{mmHg}$ or more (being higher or lower) when SMOBP and OBP were compared. This level of difference is clinically relevant and could potentially lead to different antihypertensive medication depending on how BP is recorded.

When studying potential confounders with regard to differences between OBP and SMOBP we found that the difference correlated positively with the actual OBP level. This suggests that in particular patients with a high OBP, i.e. those that are likely to be attributed to a change in medication, the SMOBP would have been considerably lower. These findings are in line with an earlier finding of a positive trend for the systolic BP difference between automated readings at the office and awake ambulatory BP reaching $78 \mathrm{mmHg}$ in the upper 95\% confidence interval [12]. We also found that smokers had a larger difference between OBP and SMOBP than nonsmokers. This difference was clinically relevant, amounting to $7.7 \pm 13$ / 3.8 \pm 4.6 $\mathrm{mmHg}$ on average, however the sub-group of current smokers was of a limited size. 
Thus knowledge about smoking habits is of importance when interpreting BP measured in these different settings. Somewhat speculatively it is possible that the higher levels of OBP relative to SMOBP in smokers could be a specific white-coat effect that is linked with the smoking habit itself, inducing an uncomfortable sense of being in an environment in which anti-smoking advices are common. Having the habit to smoke could increases BP due to worry of getting criticized for this particular life-style. In fact, the particular room used for SMOBP often is equipped with visible anti-smoking pamphlets as this room contains general information how to improve different health aspects. The comparatively small difference between SMOBP and HBP in smokers could be a consequence of increases in BP at home following the acute BP elevation induced by smoking a cigarette [13]. It is also possible that smoking frequently occurred before HBP and OBP recordings, and thus increased BP levels at these instances. Our results of lower HBP than SMOBP in general were at variance with a trial by Myers et al. of three oscillometric automatic BP recorders designed for use at home in which similar levels of BP were found in both environments [14]. However, smoking prevalence was not reported in this study by Myers et al, and if it was high this would fit with our finding of similar levels of HBP and SMOBP in smokers.

In contrast to earlier investigations on the topic our cohort included normotensive as well as hypertensive participants. However, the difference between BP measured in the different settings did not differ in hypertensive and normotensive subjects and this makes it less likely that it was better compliance with antihypertensive drug therapy that reduced HBP during seven days as compared with BP measured in the office. A limitation in this regard was that we did not measure compliance in the trial. 
Traditional office BP measured with manual technique have been shown to be lower when recorded by specially trained staff during clinic studies as compared with measurements in routine practice $[8,15]$. To take extra precaution in measurement technique in trials, when being observed, compared with routine practice has been termed the "Hawthorne effect" $[16,17]$. Since the measurement technique, the BP recorder, in our study was identical in the two settings, our results of similar BP whether measured by SMOPB or OBP suggests that the higher BP recorded manually in other studies could to some extent be dependent on lack of expertise. Thus our findings of that there in general is no increase in BP when the same technical automated equipment is used by a doctor or a nurse when compared with selfmeasurement is in line with the fact that technical issues can affect, i.e. increase, recorded blood pressure levels during routine manual recordings [16].

A limitation for the generalizability of the results was that subjects were of only moderate obesity and that hence none had arm circumference above $42 \mathrm{~cm}$. Further limitations were that we did not obtain traditional auscultatory readings for comparisons and that patients made manual notes of the self-measured BP. However, this again was based on the idea to study SMOBP and HBP in the same manner as often is the case clinically in Sweden. Also we acknowledge that results in a trial such as the one presented here were performed with "research quality" and hence that different results might be found under regular clinic conditions.

In summary our study confirms previous investigations of the importance of the setting rather than the technique, including personnel, for the BP recordings. Self- 
measurement of BP in the office with a semi-automatic device does not preclude a substantial increase in BP when levels in the individual patient is compared with selfmeasurement at home using the same equipment as illustrated with histograms as well as Bland-Altman plots Figures 1 and 2). Our data would support the use of HBP for follow-up of BP in patients with hypertension, and this is line with the fact that HBP is a better predictor of risk than is office BP measured with conventional technique [18]. The semi-automatic Omron i-C10 device did not eliminate the WCE in the same manner as do fully automated devices designed specifically for professional used such as the BpTRU, Omron 907 and Microlife WatchBP Office. A manual BP performed according to guidelines is just as good as BPs taken in the office/clinic either by a patient or health professional using a semi-automatic device, but neither technique eliminates the white-coat effect.

\section{Acknowledgements}

The study was supported by Linköping University and the County Council of Östergötland, Sweden.

\section{Duality of interest}

The authors declare that there is no duality of interest associated with this manuscript. 


\section{References}

1. Verdecchia P, Schillaci G, Borgioni C, Ciucci A, Porcellati C. White-coat hypertension. Lancet 1996; 348: 1444-6

2. Nystrom F, Aardal E, Ohman KP. A population-based study of the white-coat blood pressure effect: positive correlation with plasma cortisol. Clin Exp Hypertens 1998; 20: 95-104

3. Flynn C, Bakris GL. Role of ambulatory blood pressure monitoring in hypertension and diabetes. Curr Hypertens Rep 2013; 15: 137-42

4. Mancia G, Fagard R, Narkiewicz K, Redon J, Zanchetti A, Bohm M, et al. 2013 ESH/ESC Guidelines for the management of arterial hypertension: The Task Force for the management of arterial hypertension of the European Society of Hypertension (ESH) and of the European Society of Cardiology (ESC). J Hypertens 2013; 31:

\section{$1281-357$}

5. Myers MG, Godwin M, Dawes M, Kiss A, Tobe SW, Grant FC, et al. Conventional versus automated measurement of blood pressure in primary care patients with systolic hypertension: randomised parallel design controlled trial. BMJ 2011; 342: $\mathrm{d} 286$

6. Myers MG, Godwin M. Automated office blood pressure. Can J Cardiol 2012; 28: $341-6$

7. Stergiou GS, Efstathiou SP, Alamara CV, Mastorantonakis SE, Roussias LG. Home or self blood pressure measurement? What is the correct term? J Hypertens 2003; 21: 2259-64

8. Myers MG, Kaczorowski J, Dawes M, Godwin M. Automated office blood pressure measurement in primary care. Can Fam Physician 2014; 60: 127-32 
9. El Feghali RN, Topouchian JA, Pannier BM, El Assaad HA, Asmar RG, European Society of H. Validation of the OMRON M7 (HEM-780-E) blood pressure measuring device in a population requiring large cuff use according to the International Protocol of the European Society of Hypertension. Blood Press Monit 2007; 12: 173-8 10. Coleman A, Steel S, Freeman P, de Greeff A, Shennan A. Validation of the Omron M7 (HEM-780-E) oscillometric blood pressure monitoring device according to the British Hypertension Society protocol. Blood Press Monit 2008; 13: 49-54 11. Myers MG, Meglis G, Polemidiotis G. The impact of physician vs automated blood pressure readings on office-induced hypertension. J Hum Hypertens 1997; 11: $491-3$

12. Andreadis EA, Agaliotis GD, Angelopoulos ET, Tsakanikas AP, Chaveles IA, Mousoulis GP. Automated office blood pressure and 24-h ambulatory measurements are equally associated with left ventricular mass index. Am J Hypertens 2011; 24: $661-6$

13. Kim JW, Park CG, Hong SJ, Park SM, Rha SW, Seo HS, et al. Acute and chronic effects of cigarette smoking on arterial stiffness. Blood Press 2005; 14: 80-5 14. Myers MG, Valdivieso M, Chessman M, Kiss A. Can sphygmomanometers designed for self-measurement of blood pressure in the home be used in office practice? Blood Press Monit 2010; 15: 300-4

15. Head GA, Mihailidou AS, Duggan KA, Beilin LJ, Berry N, Brown MA, et al. Definition of ambulatory blood pressure targets for diagnosis and treatment of hypertension in relation to clinic blood pressure: prospective cohort study. BMJ 2010; 340: c1104

16. Myers MG, Valdivieso M, Kiss A. Use of automated office blood pressure measurement to reduce the white coat response. J Hypertens 2009; 27: 280-6 
17. Feil PH, Grauer JS, Gadbury-Amyot CC, Kula K, McCunniff MD. Intentional use of the Hawthorne effect to improve oral hygiene compliance in orthodontic patients. $J$ Dent Educ 2002; 66: 1129-35

18. Niiranen TJ, Hanninen MR, Johansson J, Reunanen A, Jula AM. Home-measured blood pressure is a stronger predictor of cardiovascular risk than office blood pressure: the Finn-Home study. Hypertension 2010; 55: 1346-51 
Table 1. Baseline characteristics of participants.

\begin{tabular}{|c|c|}
\hline Variable & \\
\hline Age (years) & $62.6 \pm 0.50$ \\
\hline Gender (n, males/females) & $79 / 83$ \\
\hline BMI $\left(\mathrm{kg} / \mathrm{m}^{2}\right)$ & $28.2 \pm 4.4$ \\
\hline Waist circumference $(\mathrm{cm})$ & $99.4 \pm 12$ \\
\hline Smoking (\%) & 10.5 \\
\hline Hypertension (\%) & 82.1 \\
\hline Statin treatment $(\%)$ & 29.6 \\
\hline ACE inhibitor $(\%)^{*}$ & 32 \\
\hline $\operatorname{ARB}(\%)^{*}$ & 42 \\
\hline Betablockade (\%)* & 32 \\
\hline Calcium channel blocker $(\%)^{*}$ & 33 \\
\hline Hydrochlorothiazide $(\%)^{*}$ & 22 \\
\hline Aldosterone antagonist or amiloride $(\%)^{*}$ & 7 \\
\hline Office BP $(\mathrm{mmHg})$ total cohort & $139.1 \pm 18 / 84.8 \pm 11$ \\
\hline Office BP $(\mathrm{mmHg})$ hypertension & $141.0 \pm 18 / 85.4 \pm 9.9$ \\
\hline Office BP $(\mathrm{mmHg})$ normotension & $130.1 \pm 17 / 82.3 \pm 15$ \\
\hline $\begin{array}{l}\text { Self-measured BP at office (mmHg) total } \\
\text { cohort }\end{array}$ & $138.0 \pm 19 / 85.9 \pm 10$ \\
\hline $\begin{array}{l}\text { Self-measured BP at office }(\mathrm{mmHg}) \\
\text { hypertension }\end{array}$ & $140.3 \pm 18 / 86.3 \pm 9.5$ \\
\hline $\begin{array}{l}\text { Self-measured BP at office }(\mathrm{mmHg}) \\
\text { normotension }\end{array}$ & $127.3 \pm 19 / 84.4 \pm 14$ \\
\hline $\begin{array}{l}\text { Morning Self-measured BP at home } \\
(\mathrm{mmHg}) \text { total cohort }\end{array}$ & $131.2 \pm 17 / 82.6 \pm 9.7$ \\
\hline $\begin{array}{l}\text { Morning Self-measured BP at home }(\mathrm{mmHg}) \\
\text { hypertension }\end{array}$ & $133.7 \pm 17 / 83.4 \pm 9.2$ \\
\hline $\begin{array}{l}\text { Morning Self-measured BP at home (mmHg) } \\
\text { normotension }\end{array}$ & $120.1 \pm 16 / 78.9 \pm 117$ \\
\hline $\begin{array}{l}\text { Evening Self-measured BP at home }(\mathrm{mmHg}) \\
\text { total cohort }\end{array}$ & $130.8 \pm 16 / 80.9 \pm 8.9$ \\
\hline $\begin{array}{l}\text { Evening Self-measured BP at home (mmHg) } \\
\text { hypertension }\end{array}$ & $133.0 \pm 15 / 81.5 \pm 8.3$ \\
\hline $\begin{array}{l}\text { Evening Self-measured BP at home ( } \mathrm{mmHg} \text { ) } \\
\text { normotension }\end{array}$ & $120.5 \pm 15 / 78.1 \pm 11$ \\
\hline $\begin{array}{l}\text { Mean Self-measured BP at home }(\mathrm{mmHg}) \\
\text { total cohort }\end{array}$ & $131.0 \pm 16 / 81.7 \pm 8.7$ \\
\hline $\begin{array}{l}\text { Mean Self-measured BP at home }(\mathrm{mmHg}) \\
\text { hypertension }\end{array}$ & $133.3 \pm 15 / 82.4 \pm 8.3$ \\
\hline $\begin{array}{l}\text { Mean Self-measured BP at home }(\mathrm{mmHg}) \\
\text { normotension }\end{array}$ & $120.3 \pm 15 / 78.5 \pm 10$ \\
\hline
\end{tabular}


* Percentage based on the patients with hypertension $(n=133)$. Abbreviations: Statin, HMG-CoA reductase inhibitor; ACE, angiotensin converting enzyme; ARB, angiotensin receptor blocker. 


\section{Figure legend}

Figure $1 \mathrm{a}-\mathrm{c}$

Frequency distribution of individual differences between self-measured systolic BP at office (SMOBP), office BP (OBP) and mean home BP (HBP) during seven days. Data are presented for 162 participants, three BP were recorded at each instance but the first BP that was recorded has been omitted out of three, and the mean value of the remaining two BP has been used in the analyses. HBP was measured three times in the morning and three times in the afternoon for seven days. Panel a shows frequencies of levels of systolic OBP minus SMOBP, b shows systolic OBP minus HBP and c displays systolic SMOBP minus HBP.

Figure $2 \mathrm{a}$ and $\mathrm{b}$

Bland-Altman plots of the relation between mean home BP (HBP) measured during seven days to the differences with self-measured systolic BP at office (SMOBP) or office BP (OBP). Data are presented for 162 participants, three BP were recorded at each instance but the first BP that was recorded has been omitted out of three, and the mean value of the remaining two BP has been used in the analyses. HBP was measured three times in the morning and three times in the afternoon for seven days. Panel a shows a plot for mean systolic HBP on the x-axis compared with systolic OBP minus systolic HBP on the $y$-axis. Panel b shows corresponding analysis of systolic SMOBP minus systolic HBP on the y-axis. 


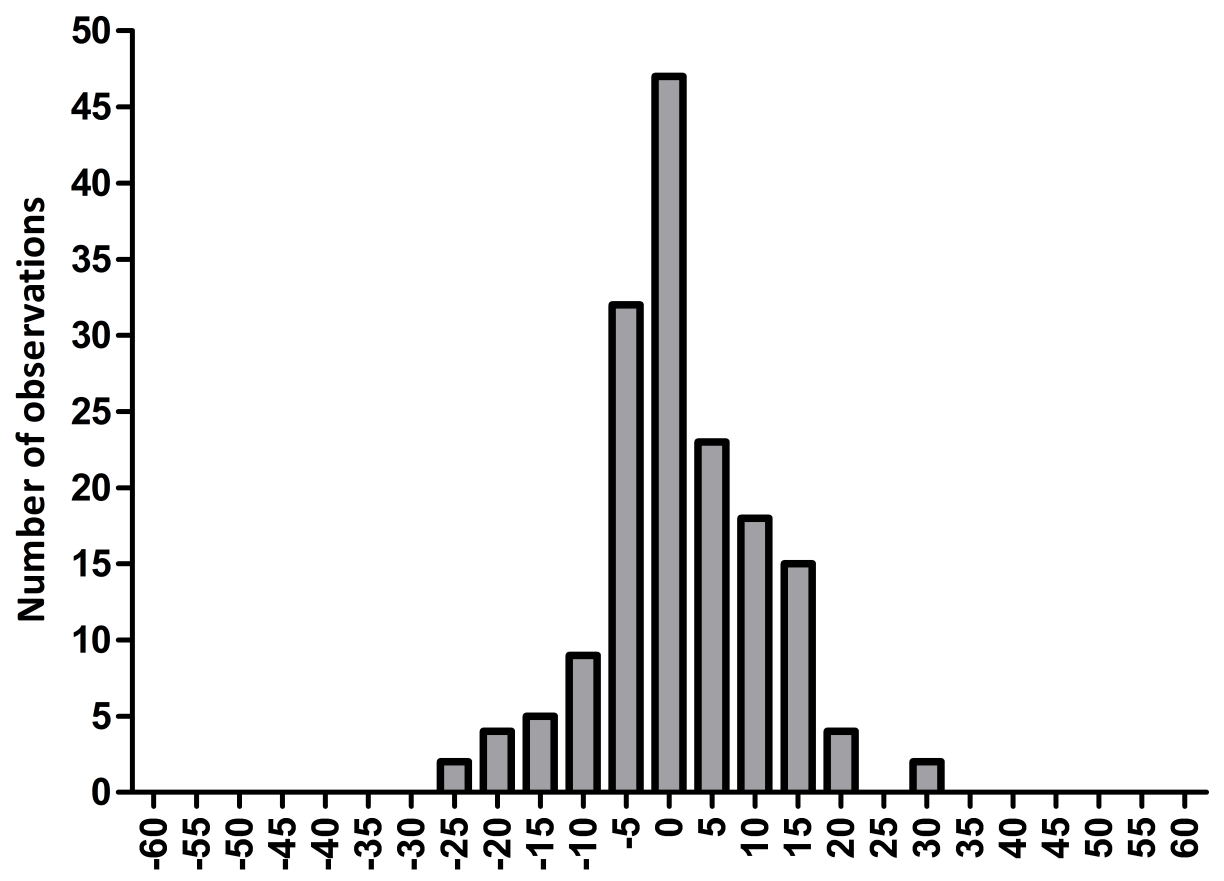

Systolic OBP minus SMOBP (mmHg) 


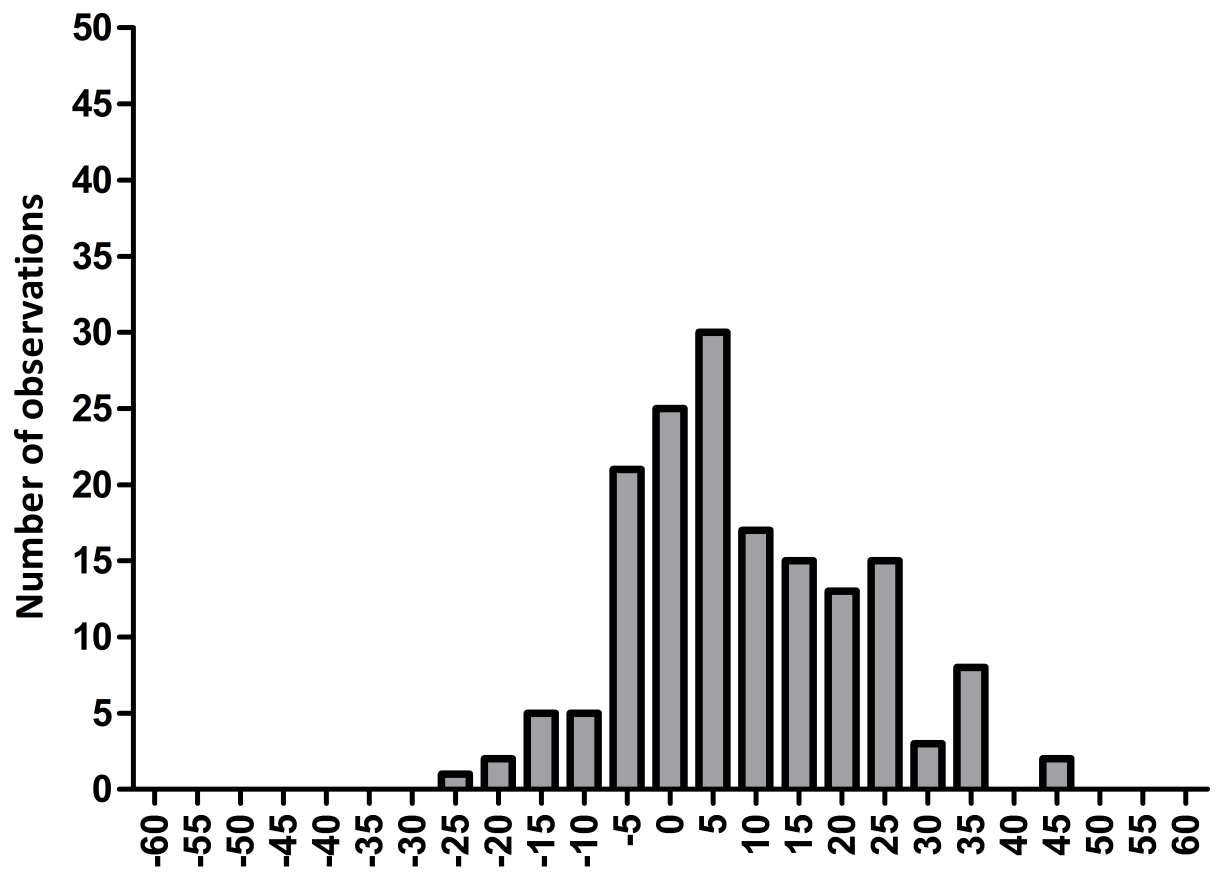

Systolic OBP minus H BP (mmHg) 


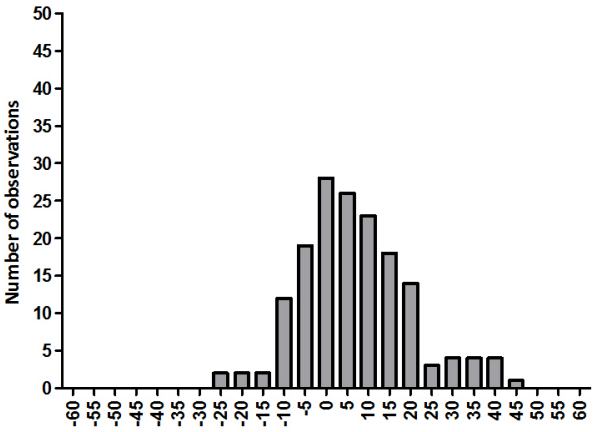

Systolic SMOBP minus H BP ( $\mathrm{mmHg}$ ) 


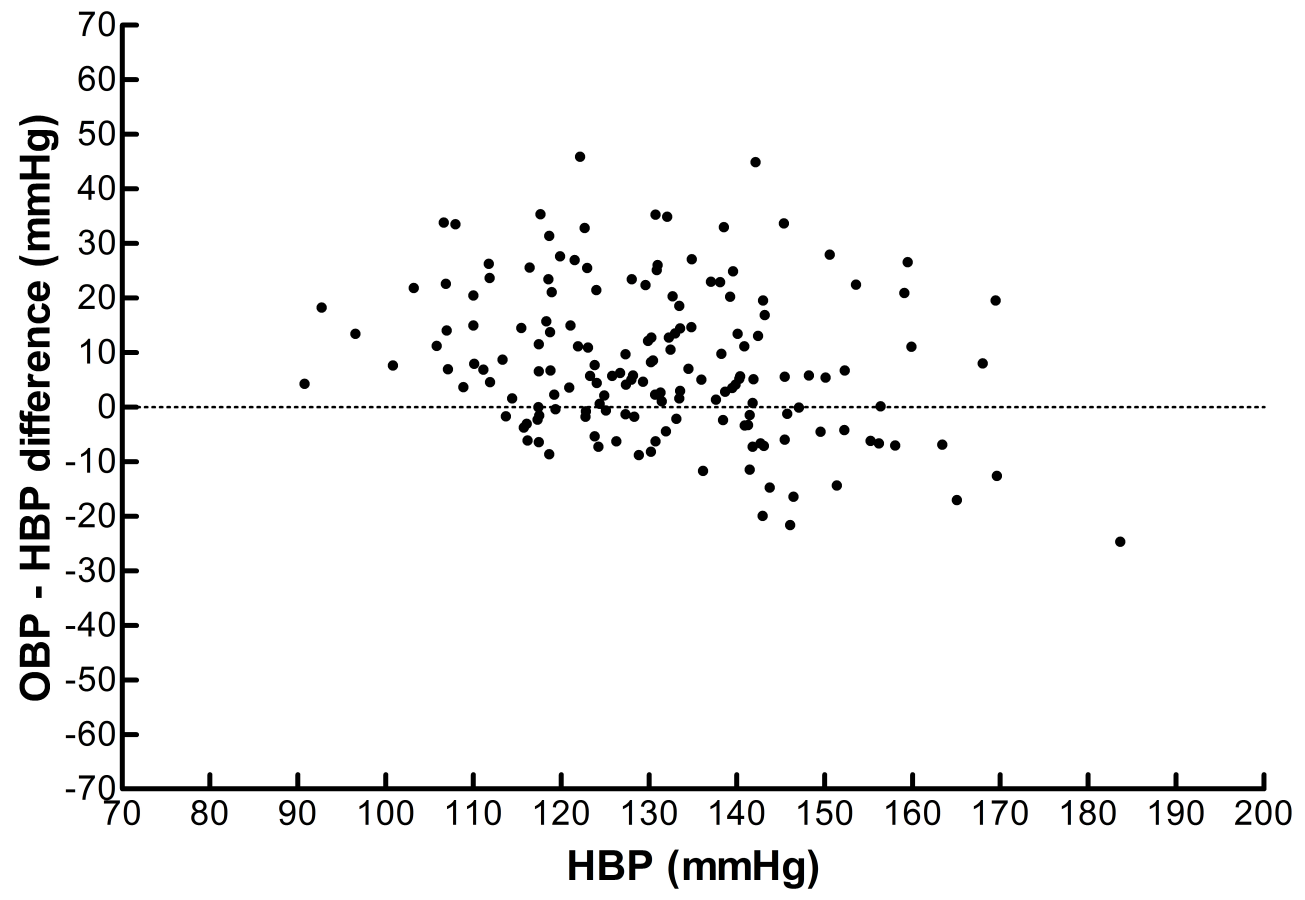




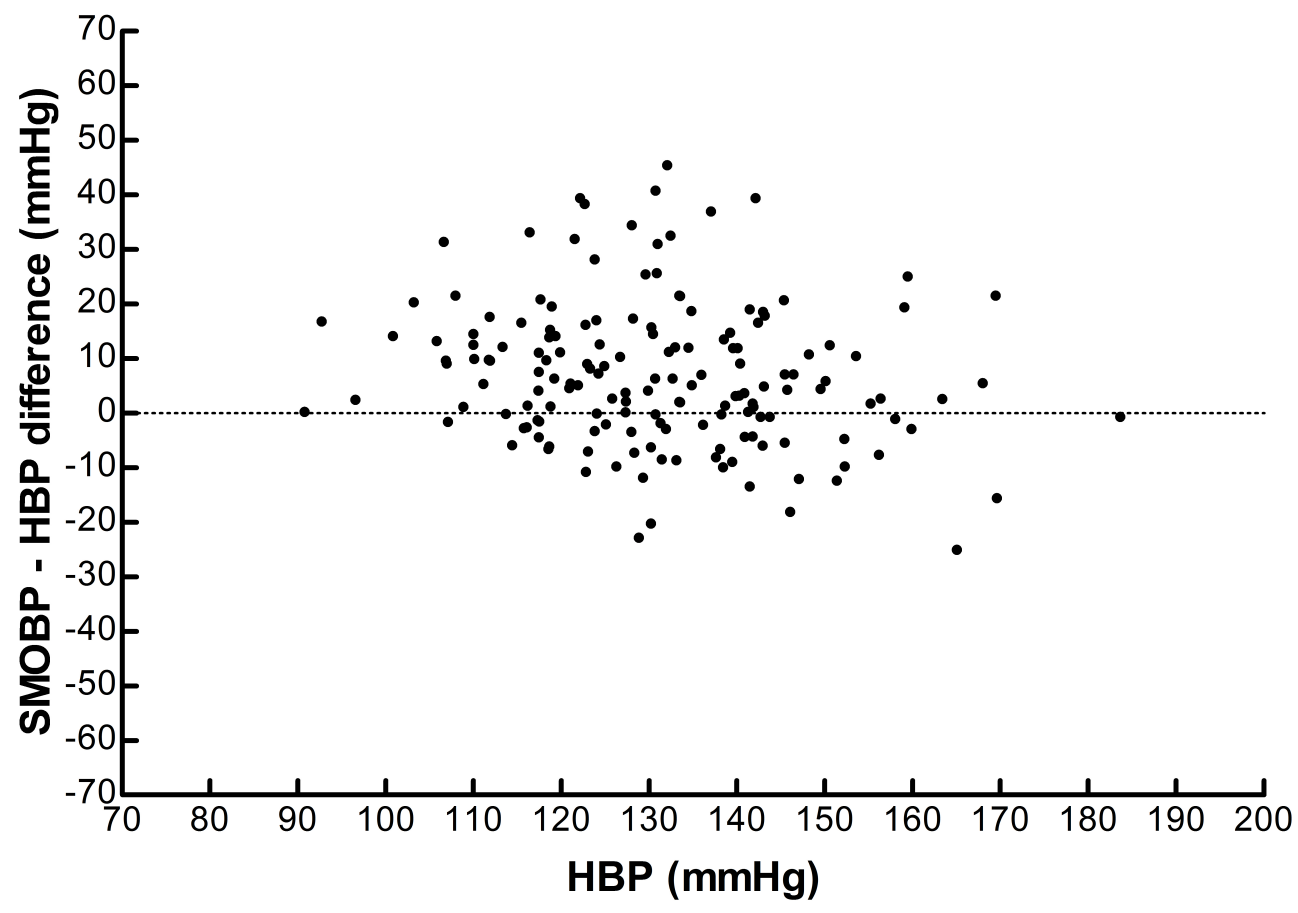

